\title{
ARTICLE Sex-dependent regulation of social reward by oxytocin receptors in the ventral tegmental area
}

\author{
Johnathan M. Borland ${ }^{1}$, Lauren M. Aiani ${ }^{1}$, Alisa Norvelle ${ }^{1}$, Kymberly N. Grantham ${ }^{1}$, Kylie O'Laughlin ${ }^{1}$, Joseph I. Terranova ${ }^{1}$,
} Kyle J. Frantz ${ }^{1}$ and H. Elliott Albers ${ }^{1}$

Social reward is critical for social relationships, and yet we know little about the characteristics of social interactions that are rewarding or the neural mechanisms underlying that reward. Here, we investigate the sex-dependent role of oxytocin receptors within the ventral tegmental area (VTA) in mediating the magnitude and valence of social reward. Operant and classical conditioning tests were used to measure social reward associated with same-sex social interactions. The effects of oxytocin, selective oxytocin receptor agonists, antagonists, and vehicle injected into the VTA on social reward was determined in male and female Syrian hamsters. The colocalization of FOS and oxytocin in sites that project to the VTA following social interaction was also determined. Females find same-sex social interactions more rewarding than males and activation of oxytocin receptors in the VTA is critical for social reward in females, as well as males. These studies provide support for the hypothesis that there is an inverted $U$ relationship between the duration of social interaction and social reward, mediated by oxytocin; and that in females the dose-response relationship is initiated at lower doses compared with males. Same-sex social interaction is more rewarding in females than in males, and an inverted $U$ relationship mediated by oxytocin may have a critical role in assigning positive and negative valence to social stimuli. Understanding these sex differences in social reward processing may be essential for understanding the sex differences in the prevalence of many psychiatric disorders and the development of gender-specific treatments of neuropsychiatric disorders.

Neuropsychopharmacology (2019) 44:785-792; https://doi.org/10.1038/s41386-018-0262-y

\section{INTRODUCTION}

Social reward plays a critical role in the formation and maintenance of almost all adaptive social relationships [1]. Indeed, the very definition of the word social can include the concept of seeking or enjoying the companionship of others, aligning with definitions of reward stimuli as those that elicit approach (although social interactions can be negative as well). Despite the fundamental importance of social reward in biological and psychological health $[2,3]$, comparatively little is known about the rewarding properties of social interactions compared to the wealth of information available on the rewarding properties of other stimuli (e.g., drugs of abuse). The relationships between the characteristics of social interactions and their rewarding properties are not well understood. For example, only recently has it been shown that there is an inverse relationship between the reward value of social interactions and the frequency of seeking those interactions [4] that is similar to the relationship between drug seeking and the reward value of drugs (e.g., cocaine dose) [5-7]. In other words, the duration of social interaction is analogous to the dose or concentration dependent effects seen with drugs of abuse. Recently, we have taken a heuristic approach to better understand social reward by developing the hypothesis that an inverted $U$ describes the relationship between the dose (i.e., duration and/or intensity) of social interaction and the reward value of those interactions [8], similar to the inverted U-shaped dose-response relationship between drug dose and reward value [9]. Initially, as the dose increases, reward value also rises, but only to a point. Once this peak is reached, increasing dose further drives down the reward value. Thus, as the duration or intensity of social interactions increase, the rewarding properties of those interactions would be initially increased, and then ultimately reduced.

The neural mechanisms mediating social reward remain to be fully defined. Recent evidence, however, indicates that the mesolimbic dopamine system, a circuit implicated in the rewarding properties of various stimuli including food, sex, and drugs of abuse, is also critical for the rewarding effects of social stimuli. Key elements in this circuit are dopamine (DA) neurons that project from the ventral tegmental area (VTA) to the nucleus accumbens (NAc). Recently, the neuropeptide oxytocin (OT), long recognized for its important role in many forms of social behavior [10], has been implicated in mediating social reward by its actions in the VTA and NAc. Inhibiting OT receptors (OTR) in the NAC in male mice [11] or in the VTA in male hamsters [12] or mice [13] significantly attenuates the rewarding properties of social interactions. Furthermore, OT fibers are in close apposition to DA neurons in the VTA that project to the NAC in male rodents [14]; OTR expression occurs in dopamine-containing neurons in the VTA [15]; and activation of OTRs in the caudal VTA leads to dopamine efflux in the NAc $[14,16]$. Taken together, these data indicate that activation of OTRs within the mesolimbic dopamine system play a critical role in mediating or encoding the rewarding properties of social interactions.

${ }^{1}$ Center for Behavioral Neuroscience, Neuroscience Institute, Georgia State University, Atlanta, GA, USA

Correspondence: H Elliott Albers (biohea@gsu.edu)

Received: 18 August 2018 Revised: 22 October 2018 Accepted: 27 October 2018

Published online: 6 November 2018 
Remarkably, nearly everything we know about the rewarding properties of social interactions and their neurobiological underpinnings have come from studies in males. Although social interactions are rewarding in both males and females [17], even the most basic questions about sex-dependent differences in the rewarding properties of social interactions have not been pursued. Here, we report the first evidence in an animal model that females find same-sex social interactions to be significantly more rewarding than males. These studies were conducted in Syrian hamsters, a species particularly well-suited for the preclinical study of behaviors that underlie psychiatric health and illness [18], and are consistent with recent evidence in humans that women find positive social interactions with same-sex partners to be more rewarding than men do [19]. Further, we test the overarching hypothesis that there is an inverted U-shaped relationship between duration of social interaction and social reward that is

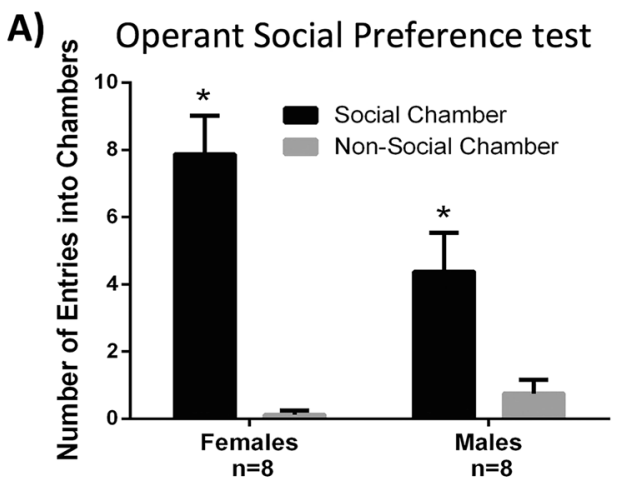

C)

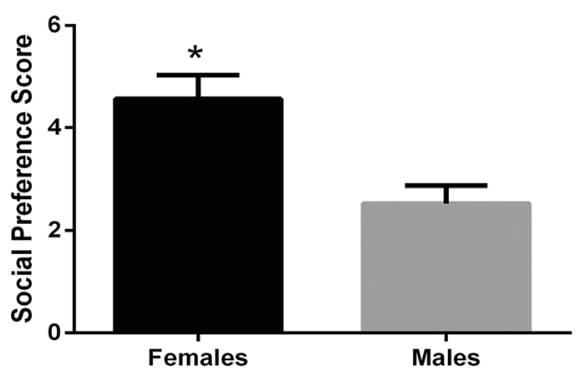

E)

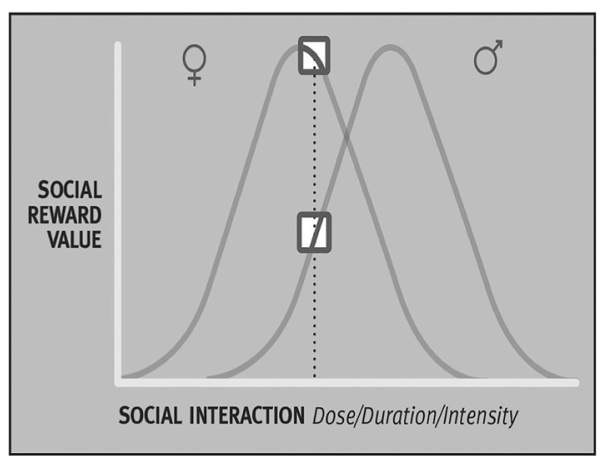

mediated by OT in both males and females, and that this dose-response relationship is initiated at lower doses in females than males [8]. Understanding the sex differences in the mechanisms of social reward is particularly important because deficits in social reward are linked with a variety of psychiatric disorders $[20,21]$, many of which are sex-dependent in terms of prevalence and predispositions, e.g., autism spectrum disorder [22].

\section{MATERIALS AND METHODS}

For complete details see the supplement.

All experiments were carried out in accordance with the National Institutes of Health Guide for the Care and Use of Laboratory Animals, and approved by the Georgia State University Institutional Animal Care and Use Committee. The rewarding

\section{B) Conditioned Place Preference test}

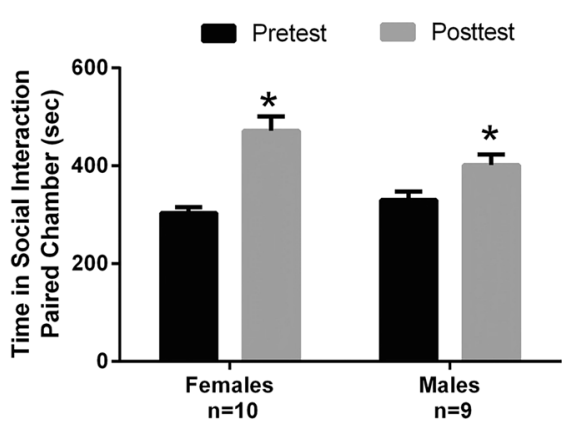

D)

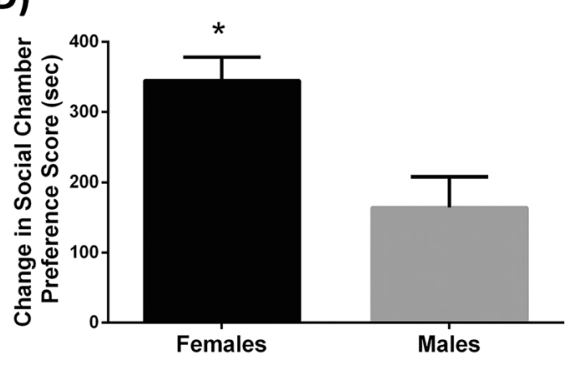

Fig. 1 Sex difference in the rewarding properties of same-sex social interaction. a Both males and females made more entries into the chambers containing same-sex stimulus hamsters compared to empty chambers in the operant social preference test. b Both males and females displayed a significant increase in the time spent in the chambers associated with same-sex social interactions during the post-test compared to the pre-test in the conditioned place preference test. c Females had a significantly higher social preference score (number of entries into the social chambers minus number of entries into the empty chambers) in the operant social preference test than males. $\mathbf{d}$ Females had a greater social preference score (time spent in the social interaction chambers minus the time spent in the no social interaction chambers) in the conditioned place preference test than males. e Illustration of the inverted $U$ hypothesis of the relationship between the dose/duration/intensity of social interactions and their reward value in males and females. In females the inverted $U$ relationship is shifted to the left relative to males because social reward is initiated at lower doses of social interaction in females than in males. As a result, social interactions that are maximally rewarding in females are only suboptimally rewarding in males. (*indicates significant difference between groups, $p<0.05)$. Error bars S.E.M 
properties of same-sex social interactions were measured in male and female Syrian hamsters using a conditioned place preference test or an operant social preference test. Females were examined daily to determine the stage of their estrous cycle by monitoring vaginal secretion. Males were also handled daily to control for any handling effects that occurred in females. Stereotaxic surgery was used to implant guide cannula in all experiments where drugs were injected into the caudal ventral tegmental area. Hamsters were allowed to recover from surgery for 4-6 days prior to behavioral testing. Following each experiment ink was injected through the guide cannula and then the site of injection was histologically evaluated. In the final experiment, immunocytochemistry and confocal microscopy was used to quantify the colocalization of FOS, a marker of neuronal activity, and oxytocin in hypothalamic neurons following a $10 \mathrm{~min}$ same-sex social interaction in males and females. Data were analyzed using SPSS software (23.0, SAS Institute, 1990) for Windows. All data were examined to determine if the assumptions of parametric analyses were met. When assumptions were violated, data were squareroot transformed. All tests were two-tailed, and results considered statistically significant if $p \leq 0.05$. All data are presented as mean \pm standard error of the mean.

\section{RESULTS}

For additional figures see the supplement.

Sex differences in the rewarding properties of social interaction In the following experiments, we investigated the rewarding properties of same-sex social interactions in male and female hamsters. Before beginning these experiments, however, we examined whether there were differences in the rewarding effects of social interactions in females at different stages of the estrous cycle, using an operant social preference test [23]. Females entered chambers containing female stimulus hamsters significantly more often than empty chambers $(p<0.001, F(1,21)=$ $46.241)$, and no significant differences were observed over the days of the estrous cycle ( $p=0.665, F(3,21)=0.533)$ (Fig. S2A, B). Although the rewarding effects of social interaction thus appeared not to change over the estrous cycle, females did display several expected estrous cycle-dependent changes in social behavior, e.g. aggression and social communication (Fig. S2C, D).
To test for sex differences in the rewarding properties of social interactions, the number of entries into a chamber containing a same-sex hamster was compared in males and females. Both males and females entered chambers containing same-sex hamsters significantly more than they entered empty chambers $(p<0.001, F$ $(1,140)=153.798)$ (Fig. 1a). Females, however, had a significantly greater social preference score (number of entries into the social chambers minus number of entries into the empty chambers) than males $(p=0.003, F(1,14)=12.615$; Fig. 1c). Indeed, the social preference score in males was $38.4 \%$ lower than in females.

Additional evidence that females find same-sex social interactions to be more rewarding than males was revealed using the conditioned place preference test. As expected, both males and females spent significantly more time in the chamber where the stimulus hamster had been paired, compared to the empty chamber $(p<0.001, F(1,17)=87.919$; Fig. 1b). Females, however, displayed a significantly greater change in the social chamber preference score compared to males $(p=0.004, F(1,17)=11.050$; Fig. 1d). The social preference score was $57.2 \%$ lower in males than in females.

To determine whether the sex differences in social place preference might result from sex differences in the characteristics of the same-sex agonistic encounters, we quantified the social behaviors that occurred during these interactions. No sex differences were observed in the duration of social investigation, aggression, grooming, or in the number of attacks or flank marks $(p>0.05)$ (Fig. S3).

OTR activation in the VTA is necessary for social reward in both males and females

We next investigated whether activation of OTRs within the VTA is necessary for social interactions to be rewarding in males and females, using the conditioned place preference test. A highly selective OTR antagonist or vehicle was injected into the caudal VTA five minutes prior to each of three social interaction conditioning sessions. The OTR antagonist significantly decreased the time spent in the chamber where the same-sex social interaction occurred in both males $(p=0.020)$ and females $(p=$ $0.003)$, compared to vehicle injected controls $(p=0.001, F(1,36)=$ 14.556) (Fig. 2b). Likewise, for the preference score, injections of the OTR antagonist decreased preference for the chamber where social interactions occurred in both males $(p=0.027)$ and females
A)

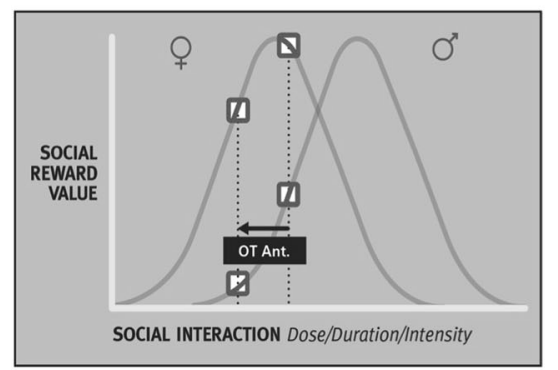

B)

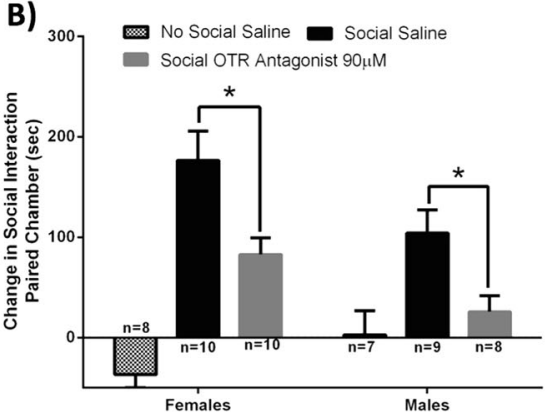

C)

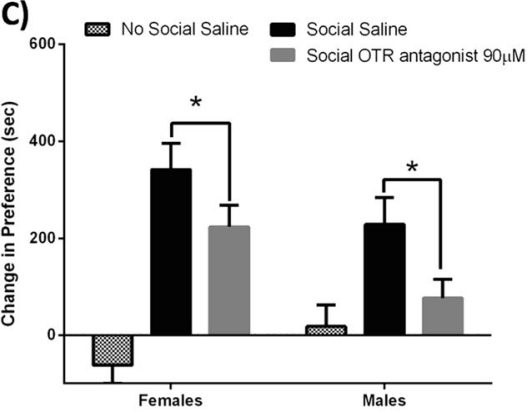

Fig. 2 Effects of inhibiting oxytocin receptor (OTR) activation in the caudal ventral tegmental area (VTA) with a highly selective OTR antagonist (Ant.) on the rewarding properties of social interaction in the conditioned place preference test. Males and females experienced same-sex social interaction in their initially non-preferred chamber and received injections of either saline or a highly selective OTR antagonist (90 $\mu \mathrm{M})$ into the caudal VTA $5 \mathrm{~min}$ prior to social conditioning sessions. a The inverted $U$ hypothesis predicts that antagonism of OTR activation reduces social reward in both males and females. b Male and female controls injected with saline in the VTA but not paired with other hamsters (i.e., No Social Saline) displayed no change in the time spent in the chambers during the post-test. In both males and females injected with saline prior to social interactions (Social Saline) there was an increase in the time spent in the social interaction paired chambers during the post-test. In both males and females, injection of the OTR antagonist into the VTA (Social OTR Antagonist) decreased the time spent in the social interaction paired chambers compared to saline injected controls during the post-test. c Male and female controls (i.e., No Social Saline) displayed no change in the social chamber preference score. In both males and females injected with saline prior to social interactions (Social Saline) there was an increase in the social chamber preference score. In both males and females, injections of the OTR antagonist into the VTA (Social OTR Antagonist) decreased the social chamber preference score compared to saline injected controls. $\left({ }^{*}\right.$ indicates significant difference between groups, $\left.p<0.05\right)$ 
A)

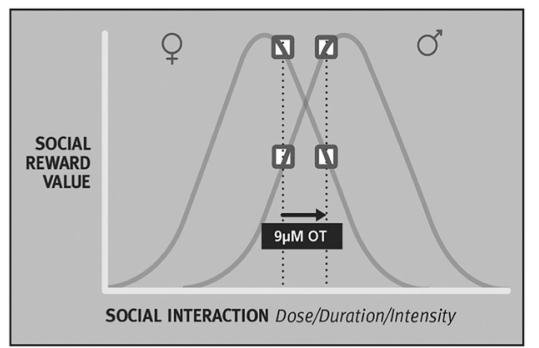

D)

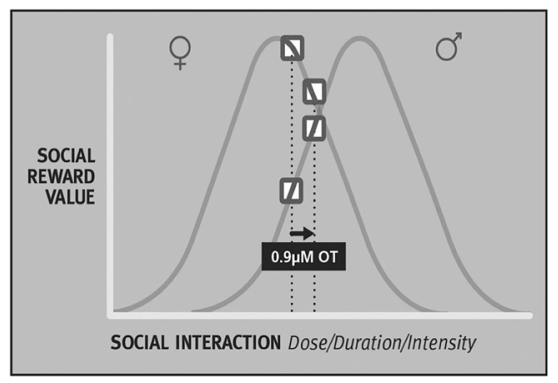

G)

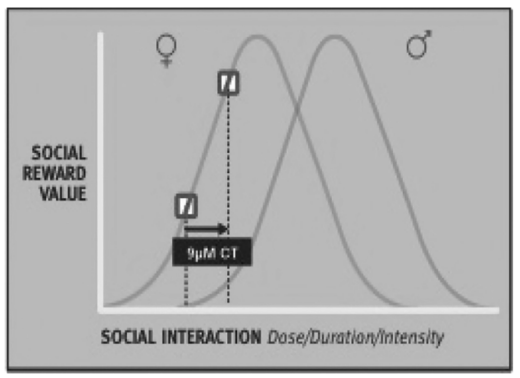

B)

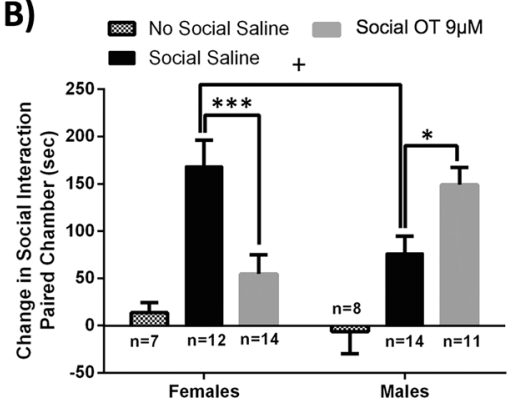

E)

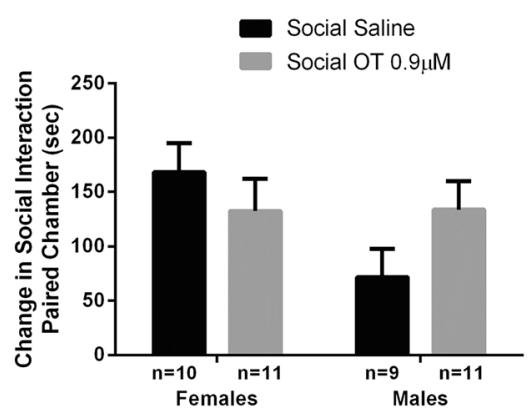

H)

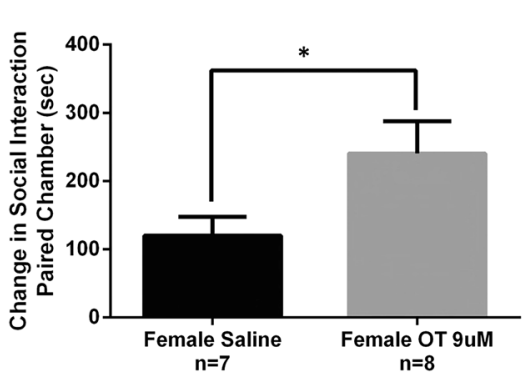

I)
C)
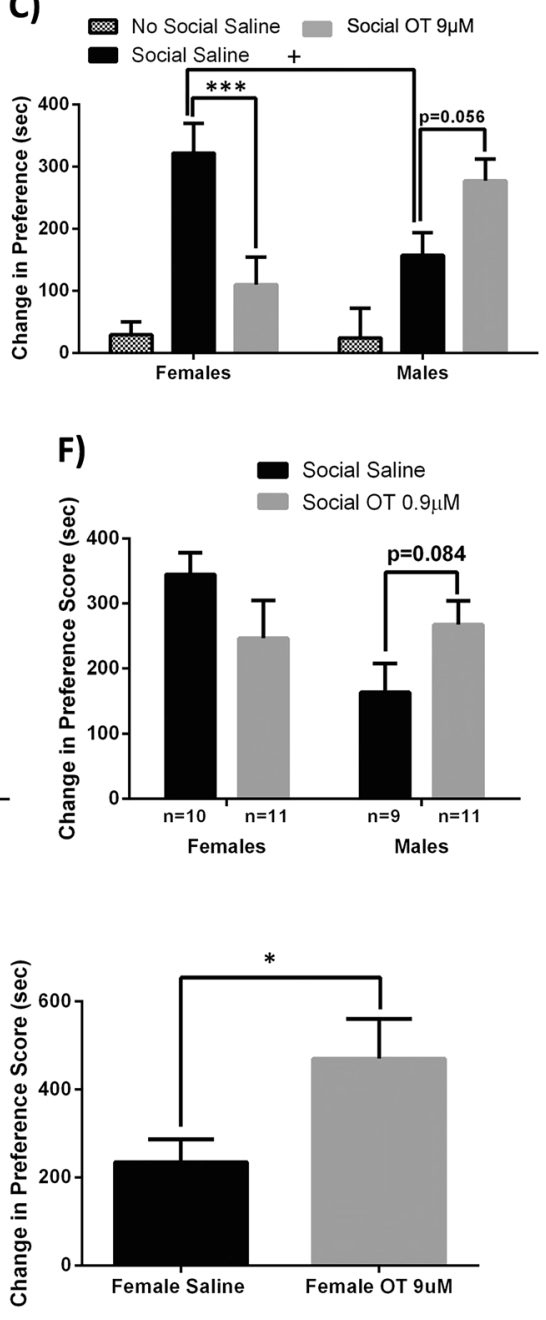

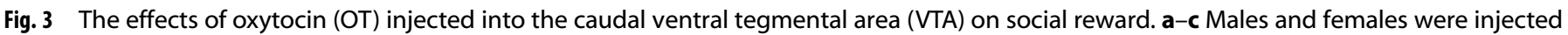

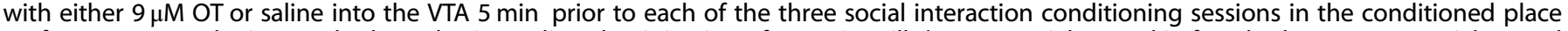

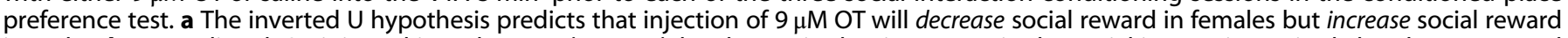

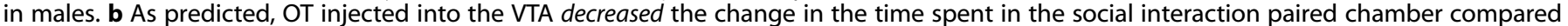

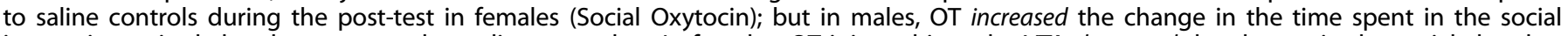

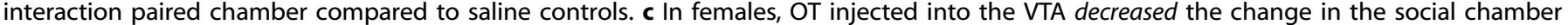

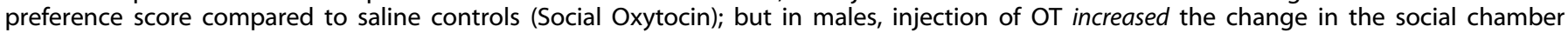

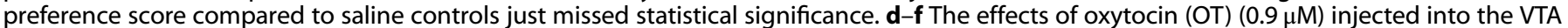

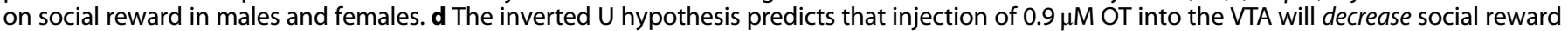

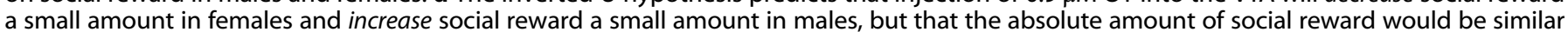

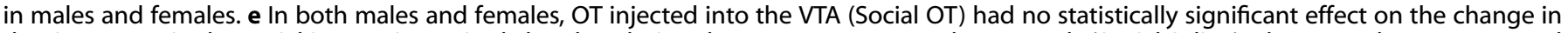

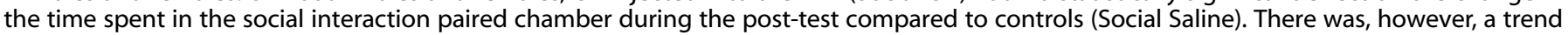

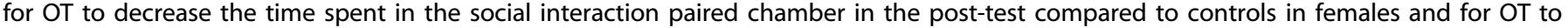

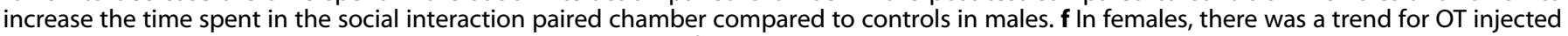

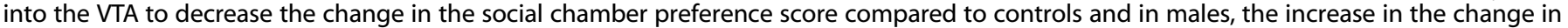

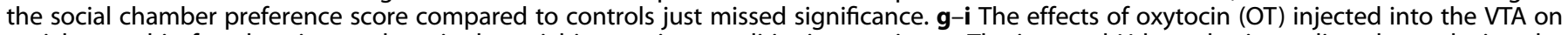

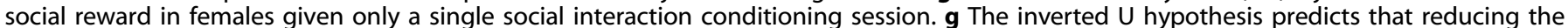

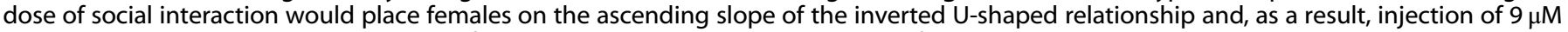

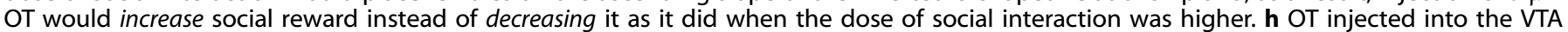

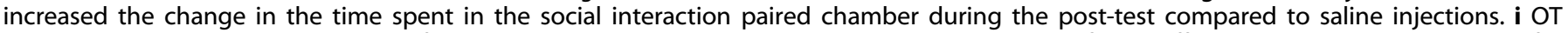

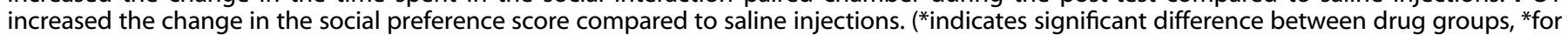
$p<0.05,{ }^{* *}$ for $p<0.01,{ }^{* * *}$ for $p<0.001 ;+$ indicates significant difference between sexes, $\left.p<0.05\right)$

$(p=0.012)$, compared to controls $(p=0.002, F(1,36)=11.221)$ (Fig. 2c). The effect size for the OTR antagonist to reduce the time spent in the social-paired chamber and social preference score was 0.306 and 0.254 , respectively. Finally, as in the previous experiments, females injected with saline spent more time in the social-paired chamber compared to males injected with saline ( $p$ $=0.024 ;$ females $176.2 \mathrm{~s}+/-29.3 ;$ males $104.1 \mathrm{~s}+/-22.7$ )
(Fig. 2b) and differences in the social chamber preference score just missed significance ( $p=0.081$; females $341.50 \mathrm{~s}+/-53.65$; males $228.51 \mathrm{~s}+/-54.94$ ) (Fig. 2c). Representative sites of injection are in Fig. S6; analysis excluded one male with the site of OTR antagonist injection outside the caudal VTA.

Again, we examined whether there were between group differences in the quality of social behavior observed during 
social pairings. The OTR antagonist in the VTA decreased the duration of social investigation in both males and females ( $p=$ $0.010, F(1,33)=7.465)$ (Fig. S4). No significant effects, nor sex differences were observed in the other behaviors measured $(p>$ 0.05).

Sex differences in the effects of intra-VTA OT on social reward Next, we tested the hypothesis that an inverted U-shaped relationship exists between the dose of social interaction and social reward, mediated by OT in both males and females, but that this dose-response relationship is initiated at lower doses in females than males, i.e., shifted leftward in females. In the first experiment, we tested the effects of $9 \mu \mathrm{M}$ OT injected into the VTA on the rewarding properties of three social interaction sessions (10 min per session) in males and females. This dose of OT was chosen because we have previously shown that it significantly increases the rewarding effects of social interactions in males [12]. We hypothesized that this "dose" of social interaction $(3 \times 10 \mathrm{~min}$ sessions) is on the ascending slope of the inverted U-shaped relationship in males. In contrast, because the inverted U-shaped relationship between the dose of social interaction and social reward is initiated at lower doses in females, we predicted that injection of this same $9 \mu \mathrm{M}$ concentration of OT into the VTA of females would significantly reduce the rewarding effects of the three sessions of social interactions by driving them into the downward slope of the inverted U (Fig. 3a). As predicted, in females, $9 \mu \mathrm{M}$ OT injected into the VTA prior to conditioning sessions decreased the time spent in the social-paired chamber $(p$ $=0.001$, OT $n=14,54.42 \mathrm{~s}+/-20.6$; saline $n=12,157.6 \mathrm{~s}+/-$ 25.0) and decreased the social chamber preference score $(p<$ 0.001 , OT $n=14,110.0 \mathrm{~s}+/-44.2$; saline $n=12,321.5 \mathrm{~s}+/-48.1)$ compared to saline controls (Fig. 3b, c). In males, however, $9 \mu \mathrm{M}$ OT in the VTA increased the time spent in the social-paired chamber $(p=0.020$, OT $n=11,149.0 \mathrm{~s}+/-18.4$; saline $n=14$, $80.2 \mathrm{~s}+/-20.1)$ and the change in social chamber preference score just missed significance $(p=0.056$, OT $n=11,277.3 \mathrm{~s}+1-$ 34.9; saline $n=14,164.1 \mathrm{~s}+/-40.6)$, compared to saline controls (Fig. 3b, c). As in the previous studies, females displayed a greater change in the time spent in the social-paired chamber than males $(p=0.008)$ and a greater change in social chamber preference score $(p=0.007)$. Again, neither sex nor OT exerted effects on aggression or social communication, although grooming differed by sex $(p<0.001,(F 1,47)=23.602)$ and OT decreased the duration of social investigation in females $(p=0.006$, OT $n=14,288.7 \mathrm{~s}$ $+/-22.3$; saline $n=12,212.5 \mathrm{~s}+/-13.8)$, but not males $(p=$ 0.404 , OT $n=11,176.4 \mathrm{~s}+/-21.5$; saline $n=14,153.5 \mathrm{~s}+/-18.4$ ) (Fig. S5). Eleven females and ten males were injected with $9 \mu \mathrm{M}$ OT outside the caudal VTA and were excluded from statistical analysis. Results from these hamsters injected with OT outside the caudal VTA (i.e., misses; Fig. S6) were similar to the results obtained from saline treated controls $(p=1.000$ for females; $p=$ 0.766 for males; data not shown).

Next, we reduced the concentration of OT injected into the VTA prior to conditioning sessions to $0.9 \mu \mathrm{M}$. Here, the inverted $\mathrm{U}$ shaped hypothesis predicted that social reward would decrease a small amount in females, increase a small amount in males, but that the absolute amount of social reward would be similar in males and females (Fig. 3d). Although there were no significant differences across groups, trends indicated that injections of 0.9 $\mu \mathrm{M}$ OT into the VTA slightly reduced both measures of social reward in females $(p=0.171, t(19)=1.422$; time in social-paired chamber: $p=0.388, t(19)=0.884$; social chamber preference score) and increased both measures in males $(p=0.084, t(18)$ $=-1.830$; time in social-paired chamber: $p=0.115$, $t(18)=-1.654$; social chamber preference score) (Fig. 3e, f). In addition, the change in the time spent in the chambers associated with social interaction $(p=0.976, t(20)=-0.305)$ and the change in the preference scores $(p=0.764, t(20)=-0.030)$ were almost identical in males and females. Investigating the effects of additional concentrations of OT was not practical, as concentrations of OT below $0.9 \mu \mathrm{M}$ are unlikely to have behavioral effects [24] and the injection of concentrations of OT above $9.0 \mu \mathrm{M}$ into the VTA induces behavioral effects that compromise the measurement of social reward [12]. In this experiment, two females and one male were excluded from statistical analysis due to injection sites outside the caudal VTA.

To further test the inverted $U$ hypothesis, we manipulated the dose of social interaction by reducing the number of $10 \mathrm{~min}$ social interaction sessions from three to one. Here, we predicted that reducing the dose of social interaction would place females on the ascending slope of the inverted U-shaped relationship and as a result injection of $9 \mu \mathrm{M}$ OT would significantly increase social reward instead of decreasing as it did when the dose of social interaction was higher (Fig. $3 \mathrm{~g}$ ). OT injected into the VTA prior to the social interaction session increased the time spent in the chamber where the social interaction occurred $(p=0.048, t(12)=$ 2.205; Fig. $3 \mathrm{~h})$ and the social chamber preference score $(p=0.044$, $t(12)=2.247$; Fig. 3i) compared to saline treated female hamsters. No subjects were excluded due to injection sites outside the caudal VTA (Fig. S6).

The effects of OT on social reward in males and females is mediated by the activation of OTRs, not vasopressin receptors The effectiveness of a highly selective OTR antagonist in the previous experiments supports the hypothesis that OTRs play an important role in mediating social reward in the VTA. However, it is unclear if the sex-specific effects of OT in the VTA on social reward are specifically mediated by activation of OTRs. Further support for the role of OTRs in mediating the effects of OT was provided in the next experiment in which a highly selective OTR agonist was injected into the VTA. An interaction of sex and drug was observed for both the time spent in the social interaction associated chamber $(p<0.001, F(1,35)=17.553)$ and social chamber preference score $(p<0.001, F(1,35)=16.664)$. As observed above with OT, in females the OTR agonist injected into the VTA decreased the time spent in the social interaction associated chamber $(p<0.001$, OTR agonist $n=10,33.8 \mathrm{~s}+/-25.5$; saline $n$ $=10176.2 \mathrm{~s}+/-29.3)$ and decreased the social chamber preference score $(p<0.001$, OTR agonist $n=10,48.8 \mathrm{~s}+/-52.3$; saline $n=10,341.5 \mathrm{~s}+/-53.6)$ compared to saline controls. In males, a trend for the OTR agonist injected into the VTA to increase the time spent in the social-paired chamber $(p=0.062$, OTR agonist $n=8,166.7 \mathrm{~s}+/-23.6$; saline $n=9,104.1 \mathrm{~s}+/-$ 22.7) and social chamber preference score was observed, compared to saline controls ( $p=0.124$, OTR agonist $n=8,333.4$ $s+/-45.9$; saline $n=9,228.5+/-54.9$; Fig. $4 a, b)$. The sites of injection in four females and nine males were outside the caudal VTA (Fig. 6S), and results from these hamsters were similar to saline controls (data not shown).

Social interaction activates OT-ir neurons in the paraventricular nucleus (PVN) and the supraoptic nucleus (SON) of males and females

The preceding experiments suggest that activation of OTRs in the VTA exerts sex-dependent effects on social reward. It is also possible, however, that sex differences in the extracellular levels OT within the VTA mediate sex differences in the rewarding properties of social interactions. One source of increased extracellular OT in the VTA may be the activation of hypothalamic magnocellular neurons. Indeed same-sex social interactions activate magnocellular neurons containing OT in the PVN of male mice [13]. To determine if sex differences in endogenous activation of OT-containing neurons occur during social interaction, we quantified the colocalization of FOS and OT in the PVN (Fig. 5a-d) and SON (Fig. 5e-h) in male and female hamsters following a 10-min social interaction. Social interaction was 

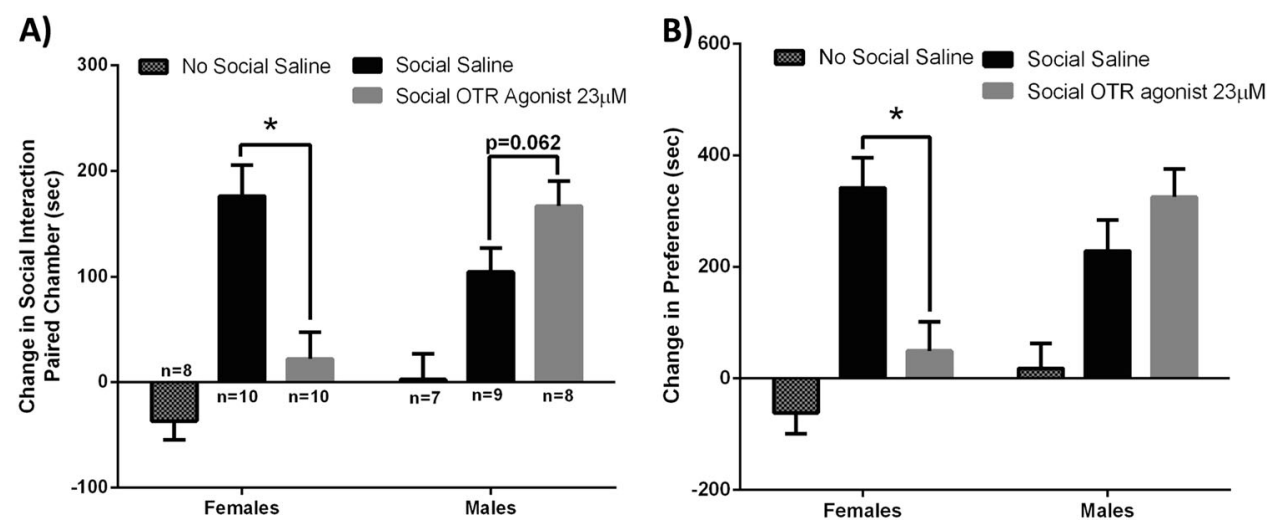

Fig. 4 The effects of injection of a highly selective oxytocin receptor (OTR) agonist into the caudal ventral tegmental area (VTA) supports the hypothesis that social reward is mediated by the activation of OT receptors (OTRs) and not vasopressin receptors in males and females. Males and females were injected with either the OTR agonist $(23 \mu \mathrm{M})$ or saline into the VTA 5 min prior to each of the three social interaction conditioning sessions in the conditioned place preference test. a Male and female controls injected with saline in the VTA but not paired with other hamsters (No Social Saline) displayed no change in the time spent in the chambers during the post-test. In both males and females injected with saline prior to social interactions (Social Saline) there was an increase in the time spent in the social interaction paired chambers during the post-test. Injections of the OTR agonist (Social OTR agonist) significantly decreased the time spent in the social interaction paired chambers during the post-test compared to saline controls in females, but in males, the OTR agonist increased the time spent in the social interaction paired chamber during the post-test although this difference just missed significance. b Male and female controls (No Social Saline) displayed no change in the social chamber preference score. Both males and females injected with saline (Social Saline) showed an increase in the social chamber preference score. Injections of the OTR agonist (Social OTR agonist) decreased the social chamber preference score compared to saline controls in females, but for males, the OTR agonist had no effect on social chamber preference score
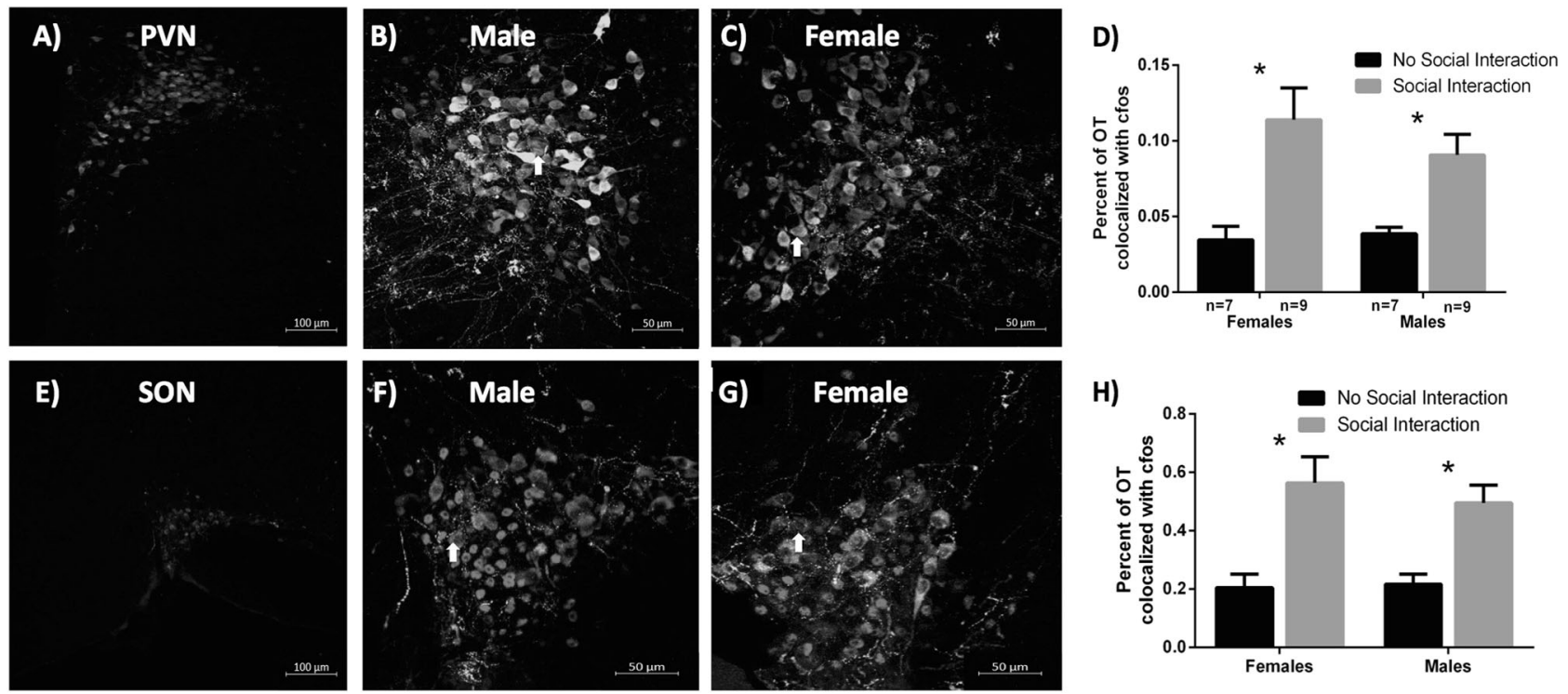

Fig. 5 Social interaction activates oxytocin (OT) immunoreactive (ir) neurons in the paraventricular nucleus (PVN) and the supraoptic nucleus (SON) of the hypothalamus in males and females. a Representative PVN image, b PVN of a male hamster, c PVN of a female hamster. $\mathbf{d}$ Both males and females show greater OT FOS colocalization in the PVN following a 10-min social interaction compared to no social interaction controls. e Representative SON image, $\mathbf{f}$ SON of a male hamster, $\mathbf{g}$ SON of a female hamster. $\mathbf{h}$ Both males and females show greater OT FOS colocalization in the SON following a 10-min social interaction compared to no social interaction controls. (*indicates significant difference between groups, $p<0.05$ )

associated with more neurons co-localized with OT and FOS in the PVN of both males and females $(p=0.037, F(1,46)=4.593)$, compared to isolated controls (Fig. 5 d). Likewise, social interaction appeared to increase activation of OT-containing neurons in the SON of males and females ( $p<0.001, F(1,47)=23.182)$ (Fig. 5h). Interestingly, a decrease in the total number of OT-containing neurons occurred in the PVN $(p=0.023, F(1,28)=5.745)$, but not the SON $(p=0.989, F(1,28)=0.000)$. No sex differences in activation of OT-containing neurons occurred in either the PVN $(p=0.217, F(1,46)=1.564)$ nor SON $(p=0.464, F(1,47)=0.544)$. No sex differences in the expression of social behavior (duration of social investigation, aggression, grooming, and frequency of flank marks and attacks) were observed (Fig. S7).

\section{DISCUSSION}

These data provide the first evidence that same-sex social interactions are more rewarding in females than in males in an animal model. This finding is consistent with studies in humans that women find positive social interactions with same-sex partners to be more rewarding than men do [19]. Further, these studies demonstrate that activation of OTRs in the VTA play a 
critical role in mediating the rewarding properties of social interactions in females as well as males. Support was also provided for the hypothesis that there is an inverted U-shaped relationship between the duration of social interaction and social reward, mediated by OT within the VTA in both males and females, and that this dose-response relationship is initiated at lower doses in females than males. As predicted, when males and females experienced the same "dose" of social interaction (i.e., $3 \times 10 \mathrm{~min}$ sessions), $9 \mu \mathrm{M}$ OT in the VTA reduced social reward in females, but increased it in males. When the dose of social interaction was reduced (i.e., one 10 min session), however, intra-VTA OT in females increased social reward. Despite these sex differences in the response to OT in the VTA, no sex differences in the number of OT-containing neurons activated by same-sex social interactions appeared in either the PVN or SON, two of the most prominent sites of OT projection into the VTA.

We have presented an inverted U-shaped hypothesis as a heuristic tool with which to consider how valence, both positive and negative, is assigned to social stimuli in a sex-dependent manner [8]. This hypothesis proposes that as the dose (i.e., duration or intensity) of social interactions increases, their rewarding nature is initially increased, but ultimately reduced. It includes the proposal that this relationship is initiated at lower intensities of social interactions in females compared to males. The utility of this hypothesis for understanding social reward will depend, at least in part, on the ability to define more clearly the critical elements of the duration or intensity of social interactions that are responsible for their rewarding properties. Parametric relationships between the duration of social interactions and its rewarding properties have been identified [4] and appear to be similar to at least some of the parametric relationships between drug dose and drug reward [5, 7]. In contrast, while the notion of an inverted $U$ relationship between the intensity of social interactions and social reward is intuitively appealing, the concept of social intensity needs to be more fully developed. For example, whether social intensity increases by the numbers of animals engaged in the interactions, the arousal levels of those animals engaged, and/or the degree of emotional involvement in the social interaction, remains unknown.

Importantly, the inverted $U$ hypothesis also proposes that the relationship between the dose of social stimuli and their reward value is mediated by the activation of OTRs in the mesolimbic dopamine system. Data consistent with an inverted $U$ dose-response relationship for the effects of OT administered systemically or directly within the VTA have been reported previously. Administration of OT into the VTA in male rats stimulates penile erections when given at intermediate doses, but not when administered at lower or higher concentrations [14]. In female mice, OT injected centrally induces a conditioned place preference for female stimulus mice at intermediate doses, but this preference is lost at higher concentrations [25]. Further support for a sex-dependent inverted $U$ dose-response relationship between OT and social reward comes from studies employing intranasal administration of OT. In female mice, pairing intranasal administration of OT $(12 \mu \mathrm{g})$ and interactions with another female mouse induces a social place preference for the stimulus mouse, but if the concentration of OT is increased to $36 \mu \mathrm{g}$, the initial preference for the female paired with OT is lost; the stimulus mouse becomes aversive [26]. In contrast, in male mice, pairing of intranasal administration of OT $(12 \mu \mathrm{g})$ with another male mouse does not induce a social place preference, thus supporting the hypothesis that the dose-response relationship between social reward and OT is initiated at higher doses in males than in females.

Studies in male rodents have established a critical role of OTRs in the VTA in activating dopamine-containing neurons [27] and in mediating social reward $[12,13]$. The mechanisms underlying the sex differences in the response to activation of OTRs are not known but may involve sex differences in the mesolimbic dopamine system (for a review ref. [28]). For example, basal extracellular levels of dopamine in the NAc are higher in female rats compared to males [29], and females display a faster rate of dopamine uptake and release than males [30]. In addition, pharmacological or electrical stimulation leads to greater dopamine efflux in the NAc of female rats compared to males $[30,31]$. Social stimuli can also trigger greater dopamine release in females than males, as shown in rats exposed to same-sex stimulus animals after a period of social isolation [32]. In female rats, the levels of the dopamine metabolite DOPAC in the striatum were higher during same-sex social interactions, whereas in males no changes in DOPAC levels were observed [33]. Similar sex differences have also been found in humans [34]. For example, women have a higher synaptic concentration of dopamine in the striatum than men [35], as well as a stronger response in the ventral striatum to prosocial decisions [36]. Therefore, it seems likely that interactions between the activation of OTRs and dopamine play a critical role in mediating the sex differences in social reward. An alternative possibility may be that the sexdependent effects of OT on social reward may be due to sex differences in neuroendocrine responses that could occur in response to aggression. For example, male and female California mice exhibit different neuroendocrine responses to aggression $[37,38]$. Yet previous studies in Syrian hamsters, although not comprehensive, have not revealed sex differences in adrenocorticotropin or cortisol levels related to agonistic encounters [39] or social isolation [40].

A key function of the social reward circuitry is the appropriate assignment of positive and negative valence to social stimuli. In many psychiatric and neurodevelopmental disorders, however, the actual or perceived valence of social stimuli normally perceived as rewarding is attenuated, and, in some cases, even reversed. The inverted $U$ hypothesis provides a simple proposal for understanding how dose of social interactions may determine reward value that can be used to investigate the mechanisms that mediate it. Further, because the inverted $U$ hypothesis proposes that the dose-response relationship between dose and social reward is initiated at lower doses in females than males, this hypothesis may provide a basis with which to investigate the substantial sex differences in many features of psychiatric and neurodevelopmental disorders. Indeed, the OTR containing circuits in the mesolimbic dopamine system may represent a target of opportunity for treating a diverse group of disorders including substance abuse, autism spectrum disorders and schizophrenia.

\section{FUNDING AND DISCLOSURE}

This work was supported by an NIH predoctoral fellowship F31MH113367 to JMB, NIH grants MH109302 and MH110212 to $\mathrm{HEA}$, and funds from the Brains and Behavior Program at Georgia State University. The authors declare no competing interests.

\section{ACKNOWLEDGEMENTS}

We thank Dr. Maurice Manning for his generous gift of OT receptor agonist and antagonist and Jason Snape for original artwork. We would also like to thank Katherine Partrick, Corey Andrews, and Asia Johnson for assisting with the experiments.

\section{ADDITIONAL INFORMATION}

Supplementary Information accompanies this paper at (https://doi.org/10.1038/ s41386-018-0262-y). 
Publisher's note: Springer Nature remains neutral with regard to jurisdictional claims in published maps and institutional affiliations.

\section{REFERENCES}

1. Trezza V, Campolongo $P$, Vanderschuren $L$. Evaluating the rewarding nature of social interactions in laboratory animals. Dev Cogn Neurosci. 2011;1:444-58.

2. Suomi SJ, Harlow HF, Kimball SD. Behavioral effects of prolonged partial social isolation in the rhesus monkey. Psychol Rep. 1971;29:1171-7.

3. Snyder-Mackler N, Sanz J, Kohn JN, Brinkworth JF, Morrow S, Shaver AO, et al. Social status alters immune regulation and response to infection in macaques. Science. 2016:354:1041-5.

4. Borland JM, Grantham KN, Aiani LM, Frantz KJ, Albers HE. Role of oxytocin in the ventral tegmental area in social reinforcement. Psychoneuroendocrinology. 2018;95:128-37.

5. Maldonado R, Robledo P, Chover AJ, Caine SB, Koob GF. D1 dopamine receptors in the nucleus accumbens modulate cocaine self-administration in the rat. Pharmacol Biochem Behav. 1993;45:239-42.

6. Veeneman MM, Broekhoven MH, Damsteegt R, Vanderschuren LJ. Distinct contributions of dopamine in the dorsolateral striatum and nucleus accumbens shell to the reinforcing properties of cocaine. Neuropsychopharmacol: Off Publ Am Coll Neuropsychopharmacol. 2012;37:487-98.

7. Doherty JM, Cooke BM, Frantz KJ. A role for the prefrontal cortex in heroinseeking after forced abstinence by adult male rats but not adolescents. Neuropsychopharmacol: Off Publ Am Coll Neuropsychopharmacol. 2013;38: 446-54.

8. Borland JM, Rilling JK, Frantz KJ, Albers HE. Sex-dependent regulation of social reward by oxytocin: an inverted $U$ hypothesis. Neuropsychopharmacol: Off Publ Am Coll Neuropsychopharmacol. 2019;44:97-110.

9. Uhl GR, Drgonova J, Hall FS. Curious cases: Altered dose-response relationships in addiction genetics. Pharmacol Ther. 2014;141:335-46.

10. Caldwell HK, Albers HE. Oxytocin, vasopressin, and the motivational forces that drive social behaviors. Curr Top Behav Neurosci. 2016;27:51-103.

11. Dolen G, Darvishzadeh A, Huang KW, Malenka RC. Social reward requires coordinated activity of nucleus accumbens oxytocin and serotonin. Nature. 2013;501:179-84.

12. Song Z, Borland JM, Larkin TE, O'Malley M, Albers HE. Activation of oxytocin receptors, but not arginine-vasopressin $\mathrm{V} 1 \mathrm{a}$ receptors, in the ventral tegmental area of male Syrian hamsters is essential for the reward-like properties of social interactions. Psychoneuroendocrinology. 2016;74:164-72.

13. Hung LW, Neuner S, Polepalli JS, Beier KT, Wright M, Walsh JJ, et al. Gating of social reward by oxytocin in the ventral tegmental area. Science. 2017;357:1406-11.

14. Melis MR, Melis T, Cocco C, Succu S, Sanna F, Pillolla G, et al. Oxytocin injected into the ventral tegmental area induces penile erection and increases extracellular dopamine in the nucleus accumbens and paraventricular nucleus of the hypothalamus of male rats. Eur J Neurosci. 2007;26:1026-35.

15. Peris J, MacFadyen K, Smith JA, de Kloet AD, Wang L, Krause EG. Oxytocin receptors are expressed on dopamine and glutamate neurons in the mouse ventral tegmental area that project to nucleus accumbens and other mesolimbic targets. J Comp Neurol. 2017;525:1094-108.

16. Shahrokh DK, Zhang TY, Diorio J, Gratton A, Meaney MJ. Oxytocin-dopamine interactions mediate variations in maternal behavior in the rat. Endocrinology. 2010;151:2276-86.

17. Douglas LA, Varlinskaya El, Spear LP. Rewarding properties of social interactions in adolescent and adult male and female rats: impact of social versus isolate housing of subjects and partners. Dev Psychobiol. 2004;45:153-62.

18. Terranova Jl, Song Z, Larkin TE 2nd, Hardcastle N, Norvelle A, Riaz A, et al. Serotonin and arginine-vasopressin mediate sex differences in the regulation of dominance and aggression by the social brain. Proc Natl Acad Sci USA. 2016;113:13233-8.
19. Feng C, Hackett PD, DeMarco AC, Chen X, Stair S, Haroon E, et al. Oxytocin and vasopressin effects on the neural response to social cooperation are modulated by sex in humans. Brain Imaging Behav. 2015;9:754-64.

20. McGregor IS, Bowen MT. Breaking the loop: oxytocin as a potential treatment for drug addiction. Horm Behav. 2012;61:331-9.

21. Foulkes L, Bird G, Gokcen E, McCrory E, Viding E. Common and distinct impacts of autistic traits and alexithymia on social reward. PLoS ONE. 2015;10:e0121018.

22. Young LJ, Pfaff DW. Sex differences in neurological and psychiatric disorders. Front Neuroendocrinol. 2014;35:253-4.

23. Borland JM, Frantz KJ, Aiani LM, Grantham KN, Song Z, Albers HE. A novel operant task to assess social reward and motivation in rodents. J Neurosci Methods. 2017;287:80-8.

24. Whitman DC, Albers HE. Role of oxytocin in the hypothalamic regulation of sexual receptivity in hamsters. Brain Res. 1995;680:73-9.

25. Kent K, Arientyl V, Khachatryan MM, Wood Rl. Oxytocin induces a conditioned social preference in female mice. J Neuroendocrinol. 2013;25:803-10.

26. Kosaki Y, Watanabe S. Conditioned social preference, but not place preference, produced by intranasal oxytocin in female mice. Behav Neurosci. 2016;130:182-95.

27. Xiao L, Priest MF, Nasenbeny J, Lu T, Kozorovitskiy Y. Biased Oxytocinergic modulation of midbrain dopamine systems. Neuron. 2017;95:368-84 e5.

28. Gillies GE, Virdee K, McArthur S, Dalley JW. Sex-dependent diversity in ventral tegmental dopaminergic neurons and developmental programing: A molecular, cellular and behavioral analysis. Neuroscience. 2014;282:69-85.

29. Virdee K, McArthur S, Brischoux F, Caprioli D, Ungless MA, Robbins TW, et al. Antenatal glucocorticoid treatment induces adaptations in adult midbrain dopamine neurons, which underpin sexually dimorphic behavioral resilience. Neuropsychopharmacol: Off Publ Am Coll Neuropsychopharmacol. 2014;39:339-50.

30. Walker QD, Rooney MB, Wightman RM, Kuhn CM. Dopamine release and uptake are greater in female than male rat striatum as measured by fast cyclic voltammetry. Neuroscience. 2000;95:1061-70.

31. Walker QD, Ray R, Kuhn CM. Sex differences in neurochemical effects of dopaminergic drugs in rat striatum. Neuropsychopharmacol: Off Publ Am Coll Neuropsychopharmacol. 2006;31:1193-202.

32. Grotewold SK, Wall VL, Goodell DJ, Hayter C, Bland ST. Effects of cocaine combined with a social cue on conditioned place preference and nucleus accumbens monoamines after isolation rearing in rats. Psychopharmacol (Berl). 2014;231:3041-53.

33. Weiss VG, Hofford RS, Yates JR, Jennings FC, Bardo MT. Sex differences in monoamines following amphetamine and social reward in adolescent rats. Exp Clin Psychopharmacol. 2015;23:197-205.

34. Mozley LH, Gur RC, Mozley PD, Gur RE. Striatal dopamine transporters and cognitive functioning in healthy men and women. Am J Psychiatry. 2001;158:1492-9.

35. Laakso A, Vilkman H, Bergman J, Haaparanta M, Solin O, Syvalahti E, et al. Sex differences in striatal presynaptic dopamine synthesis capacity in healthy subjects. Biol Psychiatry. 2002;52:759-63.

36. Soutschek A, Beharelle AR, Burke CJ, Schreiber R, Weber SC, Karipidis II, et al. The dopaminergic reward system underpins gender differences in social preferences. Nat Human Behav. 2017;1:819-27.

37. Oyegbile TO, Marler CA. Winning fights elevates testosterone levels in California mice and enhances future ability to win fights. Horm Behav. 2005;48: 259-67.

38. Trainor BC, Takahashi EY, Silva AL, Crean KK, Hostetler C. Sex differences in hormonal responses to social conflict in the monogamous California mouse. Horm Behav. 2010;58:506-12.

39. Huhman KL, Solomon MB, Janicki M, Harmon AC, Lin SM, Israel JE, et al. Conditioned defeat in male and female Syrian hamsters. Horm Behav. 2003;44: 293-9.

40. Ross AP, Norvelle A, Choi DC, Walton JC, Albers HE, Huhman KL. Social housing and social isolation: Impact on stress indices and energy balance in male and female Syrian hamsters (Mesocricetus auratus). Physiol Behav. 2017;177:264-9. 